\title{
A Virtual Backbone Construction Algorithm Based on Connected Dominating Set in Wireless Sensor Networks*
}

\author{
Shi Ting-jun", Shi Xu ${ }^{2}$ Fang Xu-ming ${ }^{1}$ \\ ${ }^{1}$ College of Information and Engineering, Yangzhou University, Yangzhou 225009, China; \\ ${ }^{2}$ School of Software Engineering, Xi'an Jiaotong University, Xi'an 710094, China \\ \{tjshi@yzu.edu.cn\}
}

\begin{abstract}
For the lack of the backbone network in wireless sensor networks, this paper proposes a virtual backbone construction algorithm based on connected dominating set in wireless sensor networks(IPCDS). The algorithm gives priority to more energy and closer nodes in the choice of backbone nodes, which makes network life longer and delay smaller.This paper proves that in unit disk graph the connected dominating set generated by the algorithm is at most 7.6opt +1.4 , the message complexity and the time complexity are $\mathrm{O}(\mathrm{n})$.Simulation results show that the IPCDS algorithm is significantly better than other algorithms.
\end{abstract}

Index Tterms - Wireless Sensor Networks, Virtual Backbone, Maximal Independent Set, Connected Dominating Set

\section{Introduction}

Wireless sensor networks have broad application prospects, uch as national security, nvironmental monitoring, raffic management, pace exploration and other fields, hus they cause the military, ndustry and academic attention. s wireless sensor networks with large-scale, elf-organization, andom deployment, omplex environment, ensor nodes with limited resources, etwork topology changes, hey often do not have a backbone network set up in advance.Under the premise of ensuring coverage and connectivity,wireless sensor networks use the connected dominating set algorithm to construct a temporary backbone network forwarding data.Only the backbone nodes are responsible for forwarding data, and those non-backbone nodes can close the communication modules to save energy. $n$ order to make the network life maximum, ireless sensor networks usually want to construct a minimal connected dominating set.However,in the unit disk graph,a minimal connected dominating set can not be completed in polynomial time [1], herefore, $\mathrm{n}$ practice, ireless sensor networks commonly use the approximation algorithm.

Based on the analysis of two classic connected dominating set algorithms, this paper proposes a virtual backbone construction algorithm based on connected dominating set(IPCDS).The algorithm constructs a backbone network with fewer nodes and smaller communication delay, and has the following advantages:a)distributed implementation;b)no positioning or orientation information;c)do not sync;d)low message and time complexity;e)high energy efficiency;f)can be extended.The rest of this article is divided into five sections.Section II is related work,section III is the IPCDS algorithms,section IV is the theoretical analysis,section V is the simulation results, the last section is the conclusion.

\section{Related Work}

At present, connected dominating set algorithms are mainly divided into two categories. The first class algorithm in the first stage solves a maximal independent set,then in the second phase select a number of connection nodes to connecte the maximal independent set.The representative of such classic algorithms is the EECDS algorithm [2].The second class algorithm in the first phase generates a non-optimal connected dominating set,and then in the second stage uses pruning rules to cut redundant leaf nodes. The representative of such classic algorithms is the CDS-Rule-K algorithm [3] [4].

The EECDS algorithm uses in the first stage the staining method for solving a maximal independent set.Initially,all nodes are dyed white.The algorithm started from a white node, while it is dyed black and broadcasts a black message. When receiving a black message,a white neighbor node was stained gray and broadcasts a gray message. When receiving a gray message,a white neighbor node broadcasts query messages to get the states and priorities of nodes around, and sets a timer.If the timer times out ago,it did not receive any black message from its adjacent nodes,then it is dyed black and broadcasts a black message,or remain white.Until all the nodes in the network were stained gray or black,the first phase of the algorithm ends.All the black nodes form a maximal independent set.The algorithm in the second phase uses the greedy manner to elect a number of connection nodes for connecting the maximal independent set.The algorithm startes from a non-independent node, while it is dyed blue and broadcast a blue message. When receiving a blue message, an independent node is dyed blue and broadcasts invitation messages. When receiving the invitation message,non-independent nodes compute the priority and broadcast update messages.A non-independent node with the greatest priority is stained blue and broadcasts a blue message.Until all the black nodes were stained blue,the second phase of the algorithm ends.All the blue nodes form a connected dominating set.

The CDS-Rule-K algorithm in the first phase uses the mark method to generate a non-optimal connected dominating set.Initially,all nodes broadcast hello messages to receive neighbor tables, and exchange their neighbor tables.If a neighbor node is not covered by other nodes, then it is marked as a node of

\footnotetext{
* This work is supported by the Natural Science Foundation of China under Grant Nos. 60803122 and 60903130.
} 
the connected dominating set.The algorithm in the second stage uses pruning rules to cut redundant leaf nodes. The pruning rule specifies that if all adjacent nodes are covered by marked brother nodes, then the node is a redundant leaf node,so it is pruned and broadcasts a updates message.Based on the above two algorithms, this paper presents networks a virtual backbone construction algorithm based on connected dominating set in wireless sensor(IPCDS).

\section{The IPCDS Algorithm}

\section{A) The concept of graph theory}

Definition1.A simple connected undirected graph:Graph $G=(V, E)$ is a simple connected undirected graph,if and only if graph $G$ satisfies three conditions:(1)any node has no loop;(2)at least one path between any two nodes;(3)at most one edge between any two nodes.where $V$ is the vertex set, $E$ is the edge set.

Definition2.Unit Disk Graph(UDG):Let graph $G$ as a simple connected undirected graph,if all nodes have the same communication radius $\mathrm{R}$, and when the distance between two nodes is not greater than $\mathrm{R}$, there exists an edge.

Definition3.Adjacent nodes:Let graph $G=(V, E)$ as the unit Disk Graph,if $u, v \in V$ and $(u, v) \in E$.

Definition4.Maximal Independent Set(MIS):Let graph $G$ $=(V, E)$ as the unit Disk Graph,if $U \subseteq V$ and $\forall u, v \in U \Rightarrow(u, v) \notin$ $E$,so $U$ is an independent set of graph $G$.if $\forall u \in(V-U) \Rightarrow U \cup\{u\}$ is not an independent set,so $U$ is a maximal independent set.

Definition5.Connected Dominating $\operatorname{Set}(\mathrm{CDS})$ :Let graph $G$ $=(V, E)$ as the unit Disk Graph,if $D \subseteq V$ and $\forall u \in(V-D) \Rightarrow(\exists v$ $\in D \cap(u, v) \in E$ ), so $D$ is a dominating set.If the sub-picture of graph $G$ derived from the $D$ is a connected graph,so $D$ is a connected dominating set.

\section{B) Algorithm description}

The IPCDS algorithm prefers more energy,large RSSI(Received Signal Strength Index) nodes to become backbone nodes, and set a timer.As the RSSI is greater, the nodes are closer. The timer value is inversely proportional to the node priority.

$$
\begin{aligned}
T_{u} & =T_{\text {con }} / W_{u} \\
W_{u} & =a \times\left(E_{u} / E_{\text {max }}\right)+b \times\left(R S S I_{u} / R S S I_{\text {max }}\right)
\end{aligned}
$$

Where $T_{u}$ is a timer value, $T_{\text {con }}$ is a time constant. $W_{u}$ is a node priority. $a$ and $b$ are the energy and distance weighting factors.If $a$ is greater than $b$, then the life are longer,or the communication delay is smaller.Eu is the residual energy of node $u, E_{\text {max }}$ is the maximum energy, $R S S I_{u}$ is the RSSI of node $u, R S S I_{\max }$ is the largest RSSI.

The IPCDS algorithm at the same stage uses the staining and markers methods to solve the maximum independent set and connected dominating set,and uses the pruning rule to further reduce the connected dominating set.Initially, all nodes are dyed white and have not been marked.The algorithm from a white node started,while it is dyed black and broadcasts a black message. When at first receiving black or gray message, a white neighbor node is marked as the child of nodes broadcasting message.The IPCDS algorithm flow is as follows:

Step1: Upon receiving a black message, if the white neighbor node is not marked,it is dyed gray and broadcasts gray messages.

Step2: Upon receiving a gray message, if the white neighbor node is not marked,then according to the formula(1) sets the timer value.If the timer times out ago,it received a black message by broadcasted the brother node,it is dyed gray and broadcasts gray messages,or it is dyed black and broadcasts black messages.

Step3: Upon receiving a black message broadcasted by the child node, he gray node was stained black and broadcasts black messages.

Step4: Upon the black node is in line with the pruning rule, it is dyed gray and broadcasts gray messages.

Until all nodes in the network are stained gray or black, the algorithm ends.All the black nodes form a connected dominating set.Figure 1 is the state diagram of the IPCDS algorithm.

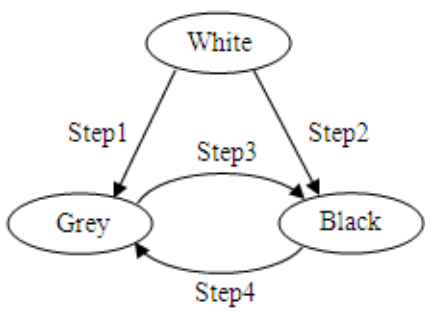

Figure 1. the state diagram of the IPCDS algorithm

\section{Theoretical Analysis}

Lemma1: The black node set generated by the algorithm IPCDS is a connected dominating set [5].

Lemma2: The connection node generated by the IPCDS algorithm Step3 connects at least two independent nodes generated by the Step2 [5].

Lemma3: In the unit disk graph, each connection node is adjacent to at most five independent nodes [6].

Lemma4: In the unit disk graph, the maximal independent set does not exceed 3.8opt +1.2 , where opt is the minimum connected dominating set [6].

Theorem1: The black node set generated by the IPCDS algorithm Step2 is a maximal independent set.

Proof:Let the black node set generated by the IPCDS algorithm Step 2 be $B$. The algorithm from the root node extends to all nodes, and dye nodes by the alternate way of a layer of gray nodes and a layer of black nodes.Except root node,each black node is dyed by the above layer of gray nodes.Black nodes increases by the2-hop distance,so they can not be adjacent.This shows that $B$ is an independent set.Until all the nodes in the tree are stained gray or black, the algorithm ends.At this time each gray node is adjacent to at least one black node,so if any one gray node will be dyed black, then $B$ will no longer be an independent set.Therefore, the black node set generated by the algorithm Step2 is a maximal independent set. 
Theorem2:The connection node set generated by the algorithm Step3 IPCDS is no more than 3.8opt +0.2 , where opt is the minimum connected dominating set.

Proof:Let $B$ be a maximal independent set, $S$ be a connection node set, $\mathrm{T}$ be a connected dominating set tree.By Lemma 2 and Lemma 3, a connection node can connect two to five independent nodes.In the worst case, all the nodes in $\mathrm{T}$ are arranged in a line.At this point,connection nodes is less than independent nodes, that $|S| \leqslant|B|-1$. By the Lemma $4, B \leqslant 3$.8opt +1.2 ,so $|S| \leqslant 3.8 \mathrm{opt}+0.2$. Therefore, the connection node set generated by the IPCDS algorithm Step3 is no more than $3.8 \mathrm{opt}+0.2$.

Theorem3: The connected dominating set generated by the IPCDS algorithm is up to 7.6 opt +1.4 , where opt is the minimum connected dominating set.

Proof: By Theorem 1 and Lemma 4, the maximal independent set produced by the IPCDS algorithm Step2 does not exceed 3.8opt +1 .2. By the theorem 2 , the connection node set produced by the IPCDS algorithm Step3 does not exceed 3.8opt +0.2 . By the Lemma 1,IPCDS algorithm generates a connected dominating set.Therefore,the connected dominating set generated by the IPCDS algorithm is up to 7.6opt +1.4 .

Theorem4: The IPCDS algorithm's message complexity and time complexity are $\mathrm{O}(\mathrm{n})$, where $\mathrm{n}$ is the network nodes.

Proof:During the algorithm Step1, each node broadcasts at most a gray message, the algorithm broadcasts at most $n$ messages.During the algorithm Step2,each node broadcasts at most a black message,the algorithm broadcasts at most $n$ messages.During the algorithm Step3, each node broadcasts at most a black message, the algorithm broadcasts at most $n$ messages.During the algorithm Step4,each node broadcasts at most a gray message,the algorithm broadcasts at most $n$ messages.In the worst case, the algorithm at most broadcasts $4 \mathrm{n}$ message,so the algorithm's message complexity is $\mathrm{O}(\mathrm{n})$.In the algorithm, the most time-consuming operation is the loop.In the worst case, the statement cycles by $\mathrm{n}$ times,so the algorithm's time complexity is $\mathrm{O}(\mathrm{n})$.

\section{Simulation Results}

The simulation assumes that all nodes are in the two-dimensional plane, and the communication radius is equal.The node has no positioning or orientation device. The initial network topology is a connected graph.In the data link layer,packet lost rate is zero.Table 1 lists all the parameters used in simulation.

Table . Simulation parameters

\begin{tabular}{|c|c|c|c|}
\hline parameter & value & parameter & value \\
\hline Range & $200 \mathrm{~m} \times 200 \mathrm{~m}$ & $E_{\max }$ & 2 Joule \\
\hline Nodes & $\begin{array}{c}10,20,40,60,80,10 \\
0\end{array}$ & $R S S I_{\max }$ & 80 \\
\hline Radius & $50 \mathrm{~m}$ & $T_{\text {con }}$ & $100 \mathrm{~ms}$ \\
\hline Weighting factor & $a=0.5, b=0.5$ & $E_{\text {elec }}$ & $50 \mathrm{~nJ} / \mathrm{bit}$ \\
\hline Message length & $50 \mathrm{byte}$ & $E_{\text {amp }}$ & $10 \mathrm{pJ} / \mathrm{bit} / \mathrm{m} 2$ \\
\hline
\end{tabular}

Figure 2 shows that when the network density increases, the backbone nodes generated by the three kinds of algorithm are increased,but the backbone nodes generated by the IPCDS algorithm are minimum.

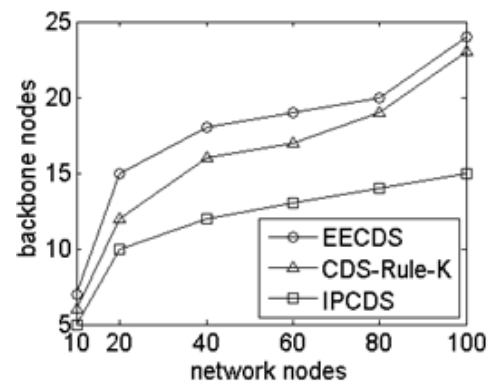

Figure 2. the backbone nodes curve with the network nodes

Figure 3 and Figure 4 show that when the network density increases, the message number and the energy consumption of the IPCDS algorithm are very slow linear increment,while those of the other algorithms are very fast non-linear increments.

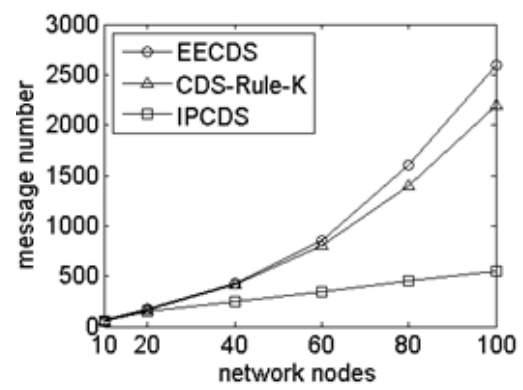

Figure 3. the message number curve with the network nodes

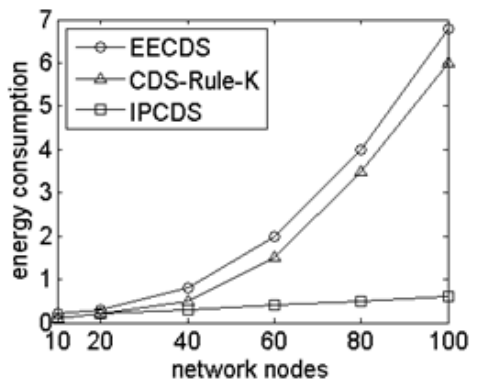

Figure 4. the energy consumption curve with the network nodes

\section{Conclusions}

This paper proposes a virtual backbone construction algorithm based on connected dominating set in wireless sensor networks(IPCDS).Theoretical analysis shows that the connected dominating set generated by the IPCDS algorithm is up to 7.6opt +1.4 , the message complexity and the time complexity are $\mathrm{O}(\mathrm{n})$.Simulation results show that IPCDS is significantly better than EECDS and CDS-Rule-K.The next step is to introduce dynamic reconfiguration technology,which makes the backbone network load more balanced and life span more extended. 


\section{References}

[1] B. N. Clack, C. J. Colbourn,D. S. Johnson.Unit Disk Graphs. Discrete Mathematics, 1990,vol.86,pp.165-177.

[2] Y. Z. Yuan, X. ia,X. H. Yan.Energy efficient distributed connected dominating sets construction in wireless sensor networks, Proceeding of the 2006 ACM International Conference on Communications and Mobile Computing, 2006,pp.797-802.

[3] J. Wu,H. Li.On calculating connected dominating set for efficient routing in ad hoc wireless networks, Proceedings of the 3rd ACM International Workshop on Discrete Algorithms and Methods for Mobile Computing and Communications, 1999,pp.7-14.

[4] J. Wu,F. Dai.An extended localized algorithm for connected dominating set formation in ad hoc wireless networks. IEEE Trans. Parallel Distributed Syst.,2004,vol.15,pp.908-920.

[5] H. Raei,M. Sarram,F. Adibniya.Optimal Distributed Algorithm for Minimum Connected Dominating Sets in Wireless Sensor Networks, Proceedings of the 5th IEEE Int'l Conference on Mobile Ad Hoc and Sensor Systems, 2008,pp.695-700.

[6] W. Wu,.H Du,.X Jia.Minimum connected dominating sets and maximal independent sets in unit disk graphs. Theoretical Computer Science, 2006, vol.352,pp.1-7. 